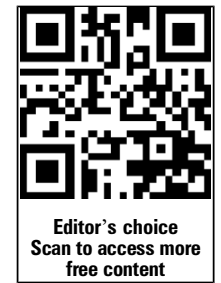

- Additional material is published online only. To view please visit the journal online (http://dx.doi.org/10.1136/ heartjnl-2013-304036).

${ }^{1}$ Department of Cardiology, Cardiovascular Research Centre, Aalborg University Hospital, Aalborg, Denmark ${ }^{2}$ Centre for Cardiovascular Sciences, University of Birmingham, City Hospital, Birmingham, UK ${ }^{3}$ Thrombosis Research Unit, Faculty of Medicine, Aalborg University, Aalborg, Denmark ${ }^{4}$ Section for Epidemiology, Department of Public Health Aarhus University, Aarhus, Denmark

\section{Correspondence to} Professor Torben Bjerregaard Larsen, Department of Cardiology, Cardiovascular Research Centre, Aalborg University Hospital, Aalborg, Denmark. Forskningens Hus, Søndre Skovvej 15, Aalborg DK-9100, Denmark; tobl@rn.dk or Professor Gregory Y H Lip, Centre for Cardiovascular Sciences, University of Birmingham, City Hospital, Birmingham B18 7QH, UK;

g.y.h.lip@bham.ac.uk

GYHL and TBL are joint senior authors.

Received 26 March 2013 Revised 23 May 2013

Accepted 24 May 2013 Published Online First 13 June 2013

\title{
Alcohol intake and prognosis of atrial fibrillation
}

\author{
Thure Filskov Overvad, ${ }^{1,2}$ Lars Hvilsted Rasmussen, ${ }^{1,3}$ Flemming Skjøth, ${ }^{1}$ \\ Kim Overvad, ${ }^{1,4}$ Ida Ehlers Albertsen, ${ }^{1,2}$ Deirdre A Lane, ${ }^{2}$ Gregory Y H Lip, ${ }^{2,3}$ \\ Torben Bjerregaard Larsen ${ }^{1,3}$
}

\section{ABSTRACT}

Objective To assess alcohol intake as a risk factor for adverse events among patients with incident atrial fibrillation (AF).

Design Prospective cohort study.

Setting Population based cohort study and nationwide Danish registries.

Patients The Danish Diet, Cancer and Health study included 57053 participants (27 178 men and 29875 women) aged between 50 and 64 years. The study population for this study included the 3107 participants (1999 men, 1108 women) who developed incident AF after inclusion.

Main outcome measures A composite of thromboembolism or death.

Results During a median follow-up of 4.9 years 608 deaths and 211 thromboembolic events occurred. Of those who developed AF, 690 (35\%) men and 233 (21\%) women had a high intake of alcohol (>20 drinks/ week for men and $>13$ drinks/week for women). After adjustment for use of oral anticoagulation and components of the $\mathrm{CHA}_{2} \mathrm{DS}_{2}$-VASc score, men with an intake of $>27$ drinks/week had a higher risk for thromboembolism or death (hazard ratio (HR) 1.33, $95 \% \mathrm{Cl} 1.08$ to 1.63$)$ than men with an intake of $<14$ drinks/week. Women with an intake of $>20$ drinks/week also had a higher risk (HR 1.23, 95\% Cl 0.78 to 1.96) than women in the low intake category. The higher risk among men was primarily driven by mortality (HR 1.51, $95 \% \mathrm{Cl} 1.20$ to 1.89 ), whereas the risk found among women was driven by thromboembolism (HR 1.71, 95\% Cl 0.81 to 3.60).

Conclusions High alcohol intake predicts thromboembolism or death, even after adjustment for established clinical risk factors, and may help identify high risk AF patients who could be targeted for stroke and cardiovascular prevention strategies.

\section{INTRODUCTION}

Atrial fibrillation (AF) is the most common sustained cardiac rhythm disorder, and is associated with a substantial risk of mortality and morbidity from ischaemic stroke and arterial thromboembolism. A variety of clinical risk stratification schemes aiming to identify high risk patients suitable for oral anticoagulation exist, the most widely used being the $\mathrm{CHADS}_{2}$ and $\mathrm{CHA}_{2} \mathrm{DS}_{2}$-VASc scores. ${ }^{1} 2$

Alcohol has been estimated to account for $3.8 \%$ of all deaths and $4.6 \%$ of all diseases and injuries worldwide. ${ }^{3}$ The intake of alcohol in light to moderate amounts is, however, associated with a lower risk of cardiovascular as well as all cause mortality, whereas heavier use is associated with a higher risk. ${ }^{4}$ The same pattern has been observed with alcohol intake and risk of stroke. ${ }^{5} 6$ A recent meta-analysis concluded that moderate to high alcohol intake is associated with a greater risk of developing AF, whereas the impact of light drinking remains uncertain. ${ }^{7}$

Alcohol use or abuse has not been determined as a risk factor for ischaemic stroke and mortality in $\mathrm{AF}$ patients. In contrast, alcohol abuse has been considered a contraindication to oral anticoagulation with the vitamin $\mathrm{K}$ antagonist (VKA) class of drugs (eg, warfarin), and alcohol intake-even in moderate amounts (eg, from $>8$ drinks per week) -has been implemented in some clinical risk scores for bleeding risk assessment among $\mathrm{AF}$ patients. $^{8-10}$

Previous studies of the association between alcohol intake and adverse events among $\mathrm{AF}$ patients have been restricted to dichotomous analyses from randomised trial cohorts, ${ }^{11}{ }^{12}$ or has been limited by few subjects with a heavy intake. ${ }^{13}$ Thus, the relationship between the full range of alcohol intake on outcomes has not been investigated prospectively with long term follow-up in a large non-selected AF cohort.

The objective of this study was to assess prospectively the associations of alcohol intake and the long term risk of thromboembolism and death among AF patients, by analysing data from the large Danish Diet, Cancer, and Health cohort study. We hypothesised that the relationship between alcohol intake and the risk of thromboembolic events and death would be either $\mathrm{J}$ or $\mathrm{U}$ shaped, with the highest risk among heavy users.

\section{METHODS}

The Diet, Cancer and Health cohort was established between 1993 and 1997, originally enrolling 57053 participants (27 178 men and 29875 women). The study design has been reported in detail elsewhere. ${ }^{14}$ Cross linkage between the cohort participants and the National Civil Registration system together with the National Patient Register ${ }^{15}$ provided detailed information on incident AF, thromboembolism, and death and specific information about censoring from emigration and death during follow-up.

\section{Incident cases of AF}

The cohort subjects were linked to the National Patient Register, dating back to 1977, using the Danish Personal Identification number. This is a

\footnotetext{
To cite: Overvad TF,
Rasmussen LH, Skjøth $F$

et al. Heart 2013;99.

1093-1099.
} 
unique and national identification number, which is part of the personal information stored in the Civil Registration System. The study population in the present study included participants who developed incident AF during follow-up.

Codes from the International Classification of Diseases, 10th revision (ICD-10) were used to extract admissions for AF. This includes all patients diagnosed with AF in hospital or ambulatories, but not from general practice. AF and atrial flutter have one common ICD-10 code (I48). Therefore, some atrial flutter cases have been included in the present study.

\section{Alcohol intake and drinking pattern}

All participants filled in a detailed semiquantitative food and drink frequency questionnaire at entry and 5 years after entry into the cohort, which has been described and validated previously. ${ }^{16} 17$ Exposure data were extracted from the questionnaire closest to but before the first diagnosis of AF. Participants reported detailed information on both frequency of intake and type of alcohol beverages consumed within the last year. The number of standard drinks per week ( $12 \mathrm{~g}$ of ethanol) was calculated on the basis of the assumed alcohol content in the different alcoholic beverages. People who reported no alcohol intake during the last year in both questionnaires were categorised as abstainers. These details were used to generate an exposure variable describing the weekly alcohol intake into five categories for women: abstainers, <7, 7-13, 13-20 and $>20$ drinks; and five categories for men: abstainers, <14, 14-20, 21-27 and $>27$ drinks.

\section{Comorbid variables}

To determine the different comorbid variables a method used by Olesen $e t$ al ${ }^{18}$ was adapted. ICD codes and ATC (Anatomical Therapeutic Chemical) drug codes used for determining the comorbid variables are available in online web-only table 1.

\section{Outcomes}

We defined our primary outcome as the composite of 'thromboembolism or death', with thromboembolism comprising ischaemic stroke and arterial thromboembolism. Secondary analyses were performed for the components of the primary composite end point, that is, 'thromboembolism' and 'death'. Information on emigration or death was available from the National Civil Registration System, and incident cases of ischaemic stroke using ICD-10 (I63.0-I63.9, I64.9) and arterial thromboembolism using ICD-10 (I74.0-I74.9) were found in the National Patient Register.

\section{Statistical methods}

Association of reported alcohol intake on risk of event was reported in terms of incidence rates for the weekly intake groups and subsequently analysed by means of Cox proportional regression using time since $\mathrm{AF}$ as the underlying time scale. End of study (30 December 2009) or emigration was considered as outcome independent censoring. For the secondary outcome event thromboembolism, death was considered as a censoring event.

All analyses were stratified by sex. The associations are reported as a crude measure of association and after adjustment for the risk factors recommended in current stroke prevention guidelines, essentially the $\mathrm{CHA}_{2} \mathrm{DS}_{2}$-VASc components. ${ }^{19-21}$ The risk factors were derived at time of $\mathrm{AF}$ diagnosis and assumed to be constant throughout time at risk. Both crude and adjusted analyses are adjusted for the effect of VKA treatment, incorporated as a time varying covariate. This was possible as all prescriptions handled in Danish pharmacies are reported to a common database. As there is no prior hypothesis on the associations of alcohol exposure in terms of the number of standard drinks per week, the effect was further modelled using a natural cubic spline and presented graphically. Data were analysed using Stata V.12 (Stata Corporation, College Station, Texas, USA). A value of $\mathrm{p}<0.05$ was considered statistically significant.

\section{RESULTS}

We identified 3366 patients with incident AF during follow-up. Of those, $259(7.7 \%)$ patients were excluded from the final analyses (figure 1), leaving a study population of 3107 cases: 1999 men and 1108 women.

Baseline characteristics according to weekly alcohol intake are presented in table 1 for men and table 2 for women. Men had a median age of 66.5 years, with a median follow-up of 4.9 years, and a median weekly alcohol intake of 12.5 drinks (10th-90th centile, 2.1-40.7) (table 1). Only 43 (2\%) males reported being abstainers and 690 (35\%) patients had a weekly alcohol intake above the Danish recommended male maximum of 21 drinks per week. Around $25 \%$ had a $\mathrm{CHA}_{2} \mathrm{DS}_{2}$-VASc score of 0 .

Women had a median age of 67.8 years, with a median follow-up of 4.7 years and a median weekly alcohol intake of 5.6 drinks per week (10th-90th centile, 0.7-21.7) (table 2). Only 45 (4\%) females reported being abstainers and 233 (21\%) reported having a weekly alcohol intake above the Danish recommended female maximum of 14 drinks per week. Around $21 \%$ had a $\mathrm{CHA}_{2} \mathrm{DS}_{2}$-VASc score of 1 (women cannot score 0 ).

\section{Follow-up}

During 17089 person years of follow-up, 608 patients died and $211 \mathrm{had}$ a thromboembolic event. Crude incidence rates for men and women are available in online web-only table 2. Hazard ratios (HRs) at full follow-up are presented in table 3 for men
Figure 1 Inclusion of the study population. CPR (unique and national identification number).

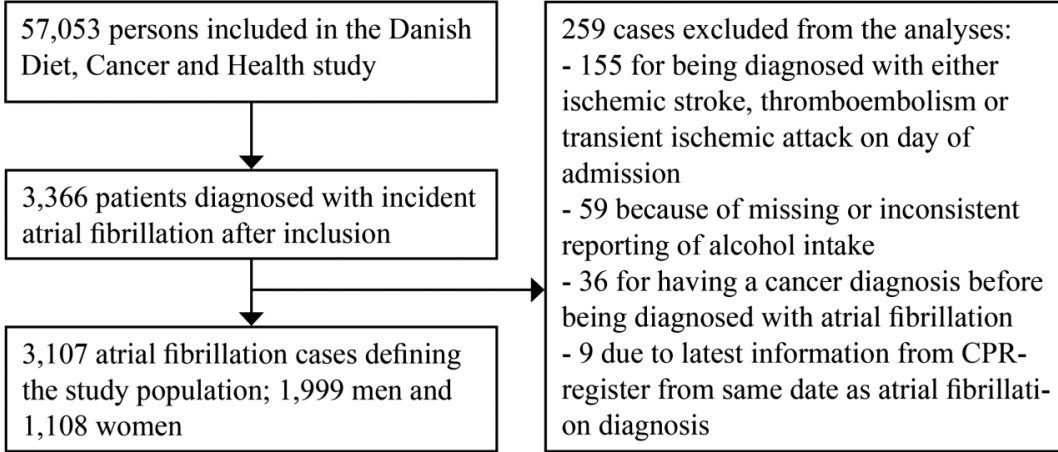


Table 1 Clinical characteristics of men according to weekly alcohol intake

\begin{tabular}{|c|c|c|c|c|c|c|}
\hline & \multicolumn{6}{|c|}{ Alcohol intake, drinks* per week } \\
\hline & All men & Abstainer & $<14$ & $14-20$ & $21-27$ & $>27$ \\
\hline Subjects, $\mathrm{n}$ & 1999 & 43 & 1025 & 241 & 234 & 456 \\
\hline Drinks per week (median, $(10 \% ; 90 \%)$ ) & $12.5(2.1-40.7)$ & N/A & $6.9(1.5-11.9)$ & $18.4(14.9-20.7)$ & $23.8(21.4-26.3)$ & $38.8(28.6-64.7)$ \\
\hline Years of follow-up (median (10\%; $90 \%)$ ) & $4.9(1.0-11.3)$ & $5.3(1.8-11.5)$ & $5.0(1.0-11.2)$ & $4.7(1.0-11.2)$ & $4.9(0.9-11.6)$ & $4.8(1.1-11.4)$ \\
\hline Age, years (median $(10 \% ; 90 \%)$ ) & $66.5(58.8-73.9)$ & $66.8(60.0-73.3)$ & $66.7(59.1-74.4)$ & $66.5(58.8-73.7)$ & $66.5(58.8-73.8)$ & $66.4(58.5-73.5)$ \\
\hline Age $\geq 75$ years & 6.7 & 4.9 & 7.7 & 6.6 & 6.0 & 5.0 \\
\hline Age $65-74$ years & 53.4 & 62.8 & 52.7 & 52.7 & 54.3 & 53.9 \\
\hline Heart failure & 7.5 & 11.6 & 8.1 & 6.2 & 5.6 & 7.2 \\
\hline Hypertension & 27.7 & 20.9 & 29.1 & 27.8 & 25.6 & 26.1 \\
\hline Diabetes & 15.3 & 18.6 & 15.0 & 17.4 & 11.5 & 16.4 \\
\hline \multicolumn{7}{|l|}{ Previous } \\
\hline Ischaemic stroke & 6.4 & 7.0 & 7.0 & 5.8 & 6.8 & 5.0 \\
\hline Transient ischaemic attack & 3.0 & 0.0 & 3.3 & 2.6 & 4.3 & 2.2 \\
\hline Arterial thromboembolism & 0.6 & 2.3 & 0.7 & 0.0 & 0.0 & 0.9 \\
\hline Haemorrhagic stroke & 1.0 & 0.0 & 0.5 & 1.7 & 0.9 & 1.5 \\
\hline \multicolumn{7}{|l|}{ Vascular disease } \\
\hline Previous myocardial infarction & 13.3 & 18.6 & 15.1 & 11.6 & 9.4 & 11.4 \\
\hline Aortic plaque & 0.4 & 2.3 & 0.4 & 0.4 & 0.0 & 0.2 \\
\hline Peripheral artery disease & 4.6 & 7.0 & 3.5 & 5.0 & 4.7 & 6.4 \\
\hline VKA treated & 21.7 & 23.3 & 21.2 & 22.8 & 20.5 & 22.8 \\
\hline $\mathrm{CHADS}_{2}$ (mean) & 0.83 & 0.81 & 0.86 & 0.82 & 0.74 & 0.81 \\
\hline 0 & 50.5 & 44.1 & 49.3 & 49.4 & 57.7 & 50.4 \\
\hline 1 & 28.0 & 34.9 & 28.2 & 31.1 & 22.2 & 28.3 \\
\hline$\geq 2$ & 21.5 & 20.9 & 22.4 & 19.5 & 20.1 & 21.3 \\
\hline $\mathrm{CHA}_{2} \mathrm{DS}_{2}-\mathrm{VAS} \mathrm{C}=0$ & 24.9 & 20.9 & 24.8 & 23.2 & 26.1 & 25.9 \\
\hline
\end{tabular}

and table 4 for women. Results for the entire cohort without stratification for sex are shown in online web-only table 3 .

Among men, the group with an intake of $<14$ drinks per week was used as reference. After multivariate analysis the highest risk of 'thromboembolism or death' among men was found among abstainers (HR 1.62, 95\% CI 0.99 to 2.66). Men with an intake of $>27$ drinks per week also had a significantly higher risk after multivariate adjustment (HR 1.33, 95\% CI 1.08 to 1.63$)$. This higher risk was primarily driven by a difference in mortality (HR 1.51, 95\% CI 1.20 to 1.89). Further adjustment for frequency of intake did not influence the results for men.

Among women, subjects with a weekly intake of $<7$ drinks was used as reference. After multivariate analysis a trend for the highest risk of 'thromboembolism or death' was found among women with an intake of 14-20 drinks per week (HR 1.25, $95 \%$ CI 0.82 to 1.92 ), which was very similar to the risk found among women in the highest intake category of $>20$ drinks per week (HR 1.23, 95\% CI 0.78 to 1.96). Abstainers had the lowest risk (HR 0.76, 95\% CI 0.38 to 1.50 ). The higher risk seen among women with an intake of 14-20 drinks per week was driven by a difference in mortality, whereas the higher risk seen among women with an intake of $\geq 20$ drinks per week was driven mainly by a difference in risk of thromboembolism, which was significant when adjusted for frequency of intake (HR 2.78, 95\% CI 1.02 to 7.60).

An analysis of the low risk subgroup of men with $\mathrm{CHA}_{2} \mathrm{DS}_{2}$-VASc $=0$ and women with $\mathrm{CHA}_{2} \mathrm{DS}_{2}$-VASc $=1$ according to alcohol intake is presented in online web-only table 4. The higher risk of thromboembolism and death was also evident in men with $\mathrm{CHA}_{2} \mathrm{DS}_{2}-\mathrm{VASc}=0$ and an intake of $>27$ drinks per week (HR 1.88, 95\% CI 1.18 to 2.99). A higher risk was again seen among the nine male abstainers, but the HR should be interpreted with caution given the small numbers. A non-significant trend was seen among the small numbers of women with $\mathrm{CHA}_{2} \mathrm{DS}_{2}-\mathrm{VAS} \mathrm{c}=1$ and an intake of $>14$ drinks per week.

In figure 2 the number of drinks per week are modelled as cubic splines. For men (figure 2A), the risk of the composite end point of thromboembolism or death was greater with higher intake of alcohol. For both secondary outcomes of 'thromboembolism' and 'death' separately, roughly similar curves were seen, although the association was stronger for death than for thromboembolism.

For women (figure 2B), the risk for the composite end point was higher with higher intake of alcohol until a peak at around 30 drinks weekly, after which the curve declined slightly. Analyses of the secondary end points of thromboembolism and death revealed that women had a higher risk for 'thromboembolism' with higher intake of alcohol in a dose-response matter, whereas the curve for 'death' rose until around 20 drinks weekly and thereafter declined to a level below one for the highest number of drinks per week.

To investigate whether the higher risk of mortality observed among men was caused by fatal bleeding events, we censored all patients with a major bleeding (see online web-only table 1 for ICD codes) followed by death within 30 days, and this did not attenuate the association (data not shown). As alcohol is also related to the development of cancer, we censored all patients who were given a cancer diagnosis during follow-up in a 
Table 2 Clinical characteristics of women according to weekly alcohol intake

\begin{tabular}{|c|c|c|c|c|c|c|}
\hline & \multicolumn{6}{|c|}{ Alcohol intake, drinks* per week } \\
\hline & All women & Abstainer & $<7$ & $7-13$ & $14-20$ & $>20$ \\
\hline Subjects, $\mathrm{n}$ & 1108 & 45 & 601 & 229 & 110 & 123 \\
\hline Drinks per week (median, $(10 \% ; 90 \%))$ & $5.6(0.7-21.7)$ & N/A & $2.3(0.6-6.2)$ & $9.3(7.4-12.2)$ & $18.8(15.0-20.2)$ & $26.5(21.7-40.9)$ \\
\hline Years of follow-up (median $(10 \% ; 90 \%)$ ) & $4.7(1.0-10.8)$ & $4.2(1.1-11.4)$ & $4.8(1.0-11.0)$ & $4.9(0.9-11.0)$ & $5.3(1.4-10.5)$ & $3.1(0.7-9.5)$ \\
\hline Age, years (median $(10 \% ; 90 \%)$ ) & $67.8(60.2-74.6)$ & $68.7(61.4-73.6)$ & $67.8(59.6-74.5)$ & $67.2(60.3-75.1)$ & $68.2(60.5-74.2)$ & $68.9(60.2-74.6)$ \\
\hline Age $\geq 75$ years & 9.1 & 8.9 & 8.7 & 10.5 & 7.3 & 10.6 \\
\hline Age $65-74$ years & 59.4 & 57.8 & 59.9 & 56.3 & 60.9 & 61.8 \\
\hline Heart failure & 5.6 & 8.9 & 8.8 & 3.1 & 5.5 & 1.6 \\
\hline Hypertension & 28.0 & 33.3 & 29.6 & 23.6 & 25.5 & 28.5 \\
\hline Diabetes & 11.4 & 26.7 & 12.1 & 9.6 & 3.6 & 12.2 \\
\hline \multicolumn{7}{|l|}{ Previous } \\
\hline Ischaemic stroke & 6.0 & 6.7 & 5.5 & 7.9 & 5.5 & 4.9 \\
\hline TIA & 3.6 & 8.9 & 4.0 & 1.3 & 2.7 & 4.9 \\
\hline Arterial thromboembolism & 0.4 & 0.0 & 0.2 & 1.3 & 0.9 & 0.0 \\
\hline Haemorrhagic stroke & 1.4 & 0.0 & 1.7 & 1.7 & 0.9 & 0.0 \\
\hline \multicolumn{7}{|l|}{ Vascular disease: } \\
\hline Previous MI & 6.5 & 4.4 & 8.0 & 5.2 & 7.3 & 1.6 \\
\hline Aortic plaque & 0.5 & 2.2 & 0.5 & 0.0 & 0.0 & 0.8 \\
\hline Peripheral artery disease & 2.7 & 2.2 & 3.0 & 1.3 & 3.6 & 3.3 \\
\hline VKA treated & 14.4 & 6.7 & 15.5 & 10.0 & 14.5 & 20.3 \\
\hline $\mathrm{CHADS}_{2}$ (mean) & 0.81 & 1.16 & 0.82 & 0.79 & 0.64 & 0.78 \\
\hline 0 & 50.8 & 37.8 & 51.1 & 52.0 & 54.5 & 48.8 \\
\hline 1 & 29.0 & 31.1 & 27.0 & 29.7 & 33.6 & 32.5 \\
\hline$\geq 2$ & 20.2 & 31.1 & 22.0 & 18.3 & 11.8 & 18.7 \\
\hline $\mathrm{CHA}_{2} \mathrm{DS}_{2}-\mathrm{VASC}=1$ & 21.3 & 17.8 & 21.1 & 22.7 & 20.9 & 21.1 \\
\hline
\end{tabular}

sensitivity analysis. The associations between alcohol intake and thromboembolism and death were still present (data not shown).

\section{DISCUSSION}

In this study we found that among men with AF, abstainers and heavy users ( $>27$ drinks per week) of alcohol had the highest risk of 'thromboembolism or death', even after adjustment for well known stroke risk factors used in current guidelines. Among women, subjects with an intake above 14 drinks per week had the highest risk of thromboembolism or death after adjustment for known stroke risk factors, whereas abstainers were not at higher risk. Our hypothesis of abstainers and heavy users having the highest risk was hereby confirmed, except abstaining women did not have a higher risk. Generally, the higher risk seen among men was mainly driven by death, whereas the higher risk among female heavy alcohol users was driven by thromboembolism. The higher risk of death found among men was not explained by either cancer or fatal bleeding. Further adjustment for frequency of intake indicated that drinking pattern was of more importance for women, as the measures of association changed for women but not for men.

\section{Strengths and limitations}

The Diet, Cancer and Health study population is a selected population where only about $35 \%$ of those invited agreed to participate. Also, we relied on hospital discharge diagnoses to diagnose AF. It is, however, unlikely that the association between alcohol intake and thromboembolism or death is systematically different between identified and non-identified participants with AF. A validation study indicated a positive predictive value of $>92 \%$ for $\mathrm{AF}$ diagnoses. ${ }^{22}$ The participants were followed in national registries with very limited loss to follow-up, and thus the study is unlikely to be subject to selection bias.

It is important to realise that this is not a study of the actual effect of alcohol per se but merely of the associations between reported alcohol intake and risk of outcomes. Due to the selfreported nature of alcohol intake, some AF patients might have been misclassified. However, this only mirrors the 'real life' clinical situation in which a physician would retrieve information about a patient's alcohol intake in a clinical history-that is, selfreported. Nonetheless, changes in drinking pattern may have occurred during follow-up, and this was not taken into account. However, we minimised this risk by assessing alcohol intake closest to, but before, first diagnosis of AF. Follow-up for vital status was almost complete, and the predictive values for stroke diagnoses is $>80 \%$ and most likely not associated with reported alcohol intake obtained from questionnaires. Information bias is thus not a likely explanation of the study results.

In the first analyses VKA treatment was taken into account, but we had no information on international normalised ratio (INR) values. The time axis was time from the diagnosis of AF and thus closely controlled for. We did not differentiate between subtypes of AF, which may have differed among alcohol subgroups. We focused on the possible added value compared to use of current guidelines, ${ }^{19-21}$ and components of the $\mathrm{CHA}_{2} \mathrm{DS}_{2}$-VASc risk score were therefore included in multivariate analyses. Heavy alcohol users are most likely also associated with other lifestyle risk factors for thromboembolism and 
Table 3 Hazard ratios $(95 \% \mathrm{Cl})$ for thromboembolism and death according to weekly alcohol intake for men with incident atrial fibrillation

\begin{tabular}{|c|c|c|c|c|c|}
\hline & \multicolumn{5}{|c|}{ Alcohol intake, drinks* per week } \\
\hline & Abstainers & $<14$ (reference) & $14-20$ & $21-27$ & $>27$ \\
\hline Subjects, n & 43 & 1025 & 241 & 234 & 456 \\
\hline \multicolumn{6}{|c|}{ Thromboembolism or death } \\
\hline VKA adjusted $\dagger$ & $1.82(1.11$ to 2.97$)$ & 1 & $0.92(0.69$ to 1.24$)$ & $1.02(0.76$ to 1.36$)$ & 1.36 (1.11 to 1.66$)$ \\
\hline Multi adjusted $¥$ & 1.62 (0.99 to 2.66$)$ & 1 & $0.99(0.74$ to 1.32$)$ & 1.11 (0.83 to 1.47$)$ & 1.33 (1.08 to 1.63$)$ \\
\hline Frequency adjusted§ & $1.28(0.75$ to 2.18$)$ & 1 & $0.97(0.70$ to 1.33$)$ & 1.07 (0.77 to 1.47$)$ & $1.26(0.98$ to 1.63$)$ \\
\hline \multicolumn{6}{|l|}{ Thromboembolism } \\
\hline VKA adjusted & 1.30 (0.47 to 3.56$)$ & 1 & $0.65(0.36$ to 1.20$)$ & $1.03(0.61$ to 1.71$)$ & 0.99 (0.66 to 1.49$)$ \\
\hline Multi adjusted & 1.19 (0.43 to 3.28$)$ & 1 & $0.72(0.39$ to 1.33$)$ & $1.08(0.65$ to 1.80$)$ & 1.02 (0.68 to 1.54$)$ \\
\hline Frequency adjusted & 0.95 (0.32 to 2.79$)$ & 1 & 0.75 (0.39 to 1.44$)$ & 1.10 (0.62 to 1.98$)$ & 1.04 (0.62 to 1.73$)$ \\
\hline \multicolumn{6}{|l|}{ Death } \\
\hline VKA adjusted & 1.16 (0.64 to 2.12$)$ & 1 & $1.03(0.72$ to 1.47$)$ & 1.03 (0.71 to 1.48$)$ & 1.45 (1.09 to 1.94$)$ \\
\hline Multi adjusted & $1.60(0.91$ to 2.81$)$ & 1 & $1.08(0.79$ to 1.49$)$ & 1.10 (0.80 to 1.52$)$ & $1.51(1.20$ to 1.89$)$ \\
\hline Frequency adjusted & 1.25 (0.69 to 2.29$)$ & 1 & $1.05(0.74$ to 1.49$)$ & 1.04 (0.73 to 1.49$)$ & 1.40 (1.06 to 1.86$)$ \\
\hline
\end{tabular}

death. These risk factors would have been potential confounders if the aim had been to specify the independent association for alcohol, but not in this study concerning a possible added value to existing risk stratification strategies. Confounding by other known stroke risk factors is therefore not an issue of concern.

Few studies have investigated the associations between reported alcohol intake and risk of thromboembolism and death in AF patients. An analysis of 2012 participants from the SPAF (Stroke Prevention in Atrial Fibrillation) I-III trials found incidence rates for ischaemic stroke to be lower with higher reported alcohol intake, and that users of alcohol had a lower risk than non-users (HR 0.4, p=0.04). ${ }^{12}$ However, dichotomisation is not discriminatory enough, and non-users may include previous users who have stopped drinking due to health issues.
Thus, using 'non-users' as reference could therefore give a biased image of the true associations, and is therefore not optimal when trying to identify high risk subjects. ${ }^{23}$ Similarly, data from the anticoagulated arms of the SPORTIF (Stroke Prevention Using Oral Thrombin Inhibitor In Atrial Fibrillation) III and IV trials found users of alcohol to have a lower risk of stroke or systemic embolism than non-users (HR $0.69,95 \%$ CI 0.51 to 0.93$).{ }^{11}$ In our sex stratified analysis, only abstaining men had a higher risk, whereas abstaining women were not at higher risk. This may reflect the fact that abstaining men could be previous heavy users of alcohol. Nonetheless, our cohort had few abstainers and change might have influenced these results. We also observed other differences between men and women, as the higher risk among heavy drinkers in men

Table 4 Hazard ratios $(95 \% \mathrm{Cl})$ for thromboembolism and death according to weekly alcohol intake for women with incident atrial fibrillation

\begin{tabular}{|c|c|c|c|c|c|}
\hline & \multicolumn{5}{|c|}{ Alcohol intake, drinks* per week } \\
\hline & Abstainers & $<7$ (reference) & 7 to 13 & 14 to 20 & $>20$ \\
\hline Subjects, $\mathrm{n}$ & 45 & 601 & 229 & 110 & 123 \\
\hline \multicolumn{6}{|c|}{ Thromboembolism or death } \\
\hline VKA adjusted $t$ & 0.90 (0.46 to 1.76$)$ & 1 & 0.94 (0.67 to 1.32$)$ & $1.20(0.78$ to 1.83$)$ & $1.08(0.68$ to 1.70$)$ \\
\hline Multi adjusted‡ & $0.76(0.38$ to 1.50$)$ & 1 & $1.06(0.74$ to 1.50$)$ & $1.25(0.82$ to 1.92$)$ & $1.23(0.78$ to 1.96$)$ \\
\hline Frequency adjusted§ & $0.70(0.35$ to 1.40$)$ & 1 & $1.30(0.85$ to 2.01$)$ & $1.66(0.96$ to 2.86$)$ & $1.71(0.93$ to 3.14$)$ \\
\hline \multicolumn{6}{|l|}{ Thromboembolism } \\
\hline VKA adjusted & 0.75 (0.18 to 3.13$)$ & 1 & $1.03(0.56$ to 1.91$)$ & $0.77(0.30$ to 1.97$)$ & $1.53(0.73$ to 3.17$)$ \\
\hline Multi adjusted & $0.66(0.16$ to 2.79$)$ & 1 & $1.14(0.61$ to 2.13$)$ & $0.77(0.301 .98)$ to & $1.71(0.81$ to 3.60$)$ \\
\hline Frequency adjusted & 0.68 (0.16 to 2.94$)$ & 1 & $1.45(0.68$ to 3.11$)$ & $1.11(0.37$ to 3.28$)$ & $2.78(1.02$ to 7.60$)$ \\
\hline \multicolumn{6}{|l|}{ Death } \\
\hline VKA adjusted & $0.84(0.39$ to 1.81$)$ & 1 & $0.93(0.63$ to 1.37$)$ & $1.24(0.77$ to 1.98$)$ & $0.97(0.57$ to 1.64$)$ \\
\hline Multi adjusted & $0.72(0.33$ to 1.55$)$ & 1 & $1.06(0.72$ to 1.57$)$ & $1.29(0.80$ to 2.08$)$ & $1.10(0.64$ to 1.89$)$ \\
\hline Frequency adjusted & $0.65(0.30$ to 1.42$)$ & 1 & 1.29 (0.79 to 2.10$)$ & 1.65 (0.89 to 3.03$)$ & 1.44 (0.72 to 2.87$)$ \\
\hline $\begin{array}{l}\text { Thromboembolism: inclu } \\
{ }^{*} \text { One drink equates to } 1 \\
\text { †Calculated using Cox's } \\
\text { łFurther adjustment for } \\
\text { §Also adjusted for frequ } \\
\text { VKA, vitamin K antagon }\end{array}$ & $\begin{array}{l}\text { emic stroke or peripher } \\
\text { e alcohol. } \\
\text { with time since atrial } \\
\text { VASc components. } \\
\text { take (not weekly, non-c }\end{array}$ & $\begin{array}{l}\text { thromboembolism. } \\
\text { diagnosis as unde } \\
\text { ily). }\end{array}$ & $\begin{array}{l}\text { of association in bold } \\
\text { ee variable and adjusted }\end{array}$ & $\begin{array}{l}\text { statistical significance. } \\
\text { treatment. }\end{array}$ & \\
\hline
\end{tabular}



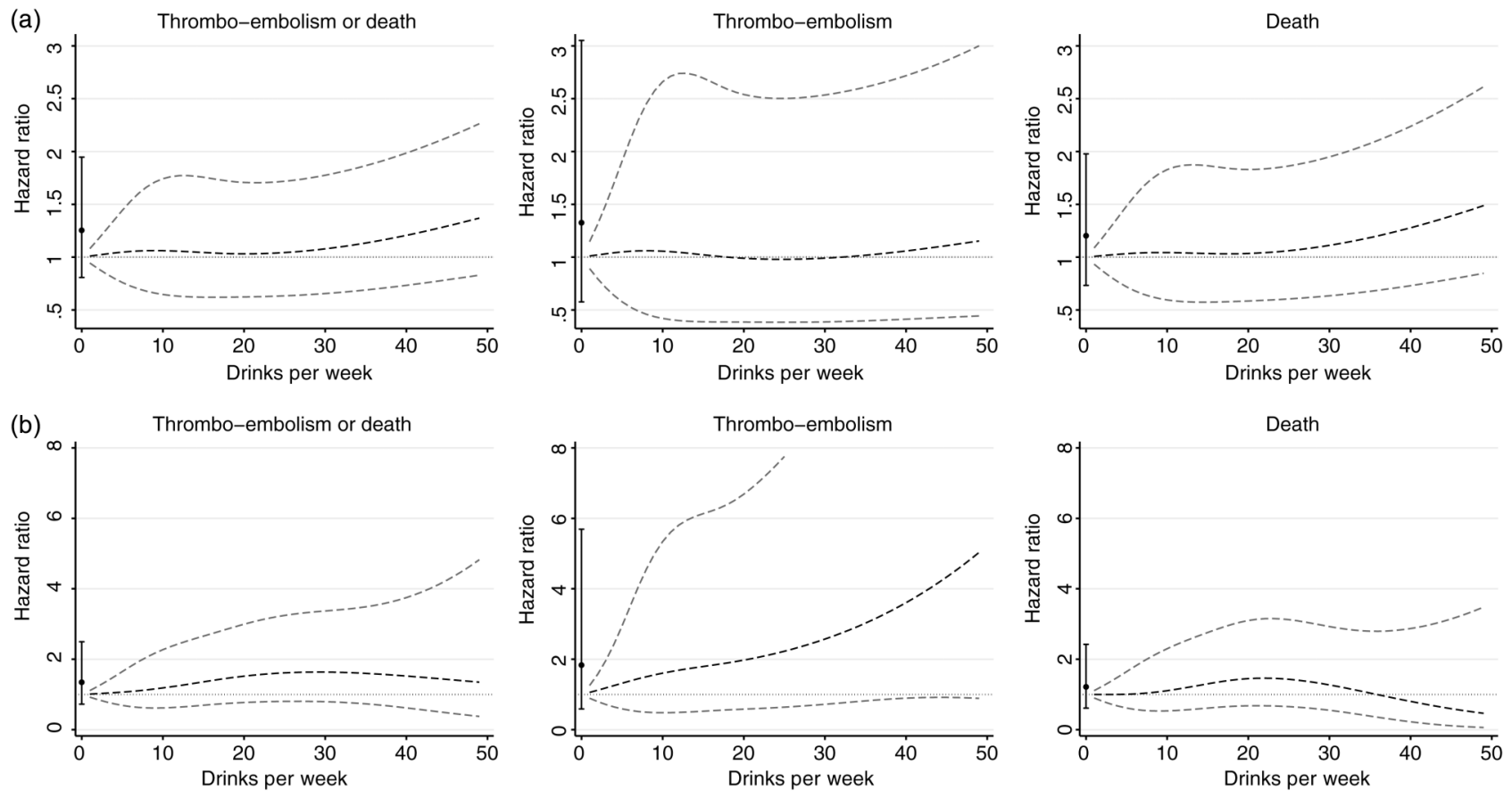

Figure 2 Continuous analysis of alcohol intake and the risk for thromboembolism and death. All analyses adjusted for vitamin $\mathrm{K}$ antagonist treatment and $\mathrm{CHA}_{2} \mathrm{DS}_{2}$-VASc components. Black dots indicate hazard ratio with $95 \% \mathrm{Cl}$ for abstainers. (a) Men. (b) Women.

was driven by mortality in contrast to thromboembolism in women. On a general population level heavy alcohol intake is associated with stroke and death among both men and women. ${ }^{6}$ Considering this is not a study of the effect of alcohol, these differences might pertain to differences in lifestyle between sexes that were not taken into account in the present analyses.

In the Cardiovascular Health Study, Mukamal and colleagues investigated the relationship between reported alcohol intake and mortality among 1232 AF patients. ${ }^{13}$ After multivariate analysis, including adjustment for concurrent warfarin treatment, all groups of alcohol users had a lower risk of death compared with never drinkers, with the highest intake category defined as $\geq 14$ drinks per week. Thus, they mainly studied the associations for moderate alcohol intake, as the average intake among incident AF cases was quite low (2.6 drinks per week against 15.0 drinks per week in the present analysis).

\section{Clinical implications}

We have chosen a composite end point including the outcomes affected by intervention with oral anticoagulation, since VKA use prevents both thromboembolism and death in AF patients. ${ }^{24}$ Importantly, some deaths in our study are likely to be due to undiagnosed ischaemic stroke caused by the register based nature of the dataset. The two most widely used stroke risk stratification schemes, $\mathrm{CHADS}_{2}$ and $\mathrm{CHA}_{2} \mathrm{DS}_{2}$-VASc, are capable of predicting both stroke and death among $\mathrm{AF}$ patients. $^{25}$

In recent years, two new oral anticoagulants have gained approval for stroke prevention in AF patients, with one additional drug nearing approval. ${ }^{26-28}$ Each has fundamentally different pharmacokinetic properties than the VKAs, and their anticoagulant effects are not directly affected by high intake of alcohol. The role of alcohol as a risk factor for bleeding in anticoagulated patients will thus potentially diminish, as the new oral anticoagulants will become more widely used. Reported alcohol intake may then help to identify subjects at high risk for ischaemic events without worrying about alcohol interfering with the anticoagulant effect of these new and safer agents. Importantly, we found indications that even among those considered low risk subjects (men with $\mathrm{CHA}_{2} \mathrm{DS}_{2}-\mathrm{VAS} \mathrm{c}=0$ and women with $\mathrm{CHA}_{2} \mathrm{DS}_{2}$-VASc=1) alcohol intake might help to differentiate them into low risk and high risk patients, but due to small numbers further studies are needed to confirm this.

Further studies are also needed to confirm our results in an AF cohort with older participants and other ethnic groups, as our cohort was relatively young and predominantly white. If confirmed, self-reported alcohol intake may help identify subjects potentially at high risk of thromboembolic events and death once diagnosed with incident AF.

\section{CONCLUSION}

High alcohol intake predicts 'thromboembolism or death', even after taking into account clinical risk factors recommended by current guidelines. High alcohol consumption may help identify AF patients with a higher risk for 'thromboembolism or death', who could be targeted for stroke and cardiovascular prevention strategies.

Contributors All authors contributed to the study design, data analysis, manuscript writing, reviewing and editing.

Competing interests DAL and GYHL are co-authors of the original $\mathrm{CHA}_{2} \mathrm{DS}_{2}$-VASC study. GYHL has served as a consultant for Bayer, Astellas, Merck, Sanofi, BMS/Pfizer, Daiichi-Sankyo, Biotronik, Portola and Boehringer Ingelheim and has served as a speaker for Bayer, BMS/Pfizer, Boehringer Ingelheim and Sanofi Aventis. DAL has received an investigator initiated educational grant from Bayer Healthcare and honoraria from Boehringer Ingelheim, Bayer Healthcare, Bristol Myers Squibb, Sanofi Aventis and Pfizer. In addition, DAL is a panellist on the 9th edition of the American College of Chest Physicians guidelines on antithrombotic therapy in AF. TBL and LHR have served as speakers for Bayer, BMS/Pfizer and Boehringer Ingelheim. TFO, FS, IEA and KO have no potential conflicts of interest to declare.

Provenance and peer review Not commissioned; externally peer reviewed.

\section{REFERENCES}

1 Gage BF, Waterman AD, Shannon W, et al. Validation of clinical classification schemes for predicting stroke: results from the National Registry of Atrial Fibrillation. JAMA 2001;285:2864-70. 
2 Lip GYH, Nieuwlaat R, Pisters R, et al. Refining clinical risk stratification for predicting stroke and thromboembolism in atrial fibrillation using a novel risk factor-based approach: the Euro Heart Survey on atrial fibrillation. Chest 2010;137:263-72.

3 Rehm J, Mathers C, Popova S, et al. Global burden of disease and injury and economic cost attributable to alcohol use and alcohol-use disorders. Lancet 2009; 373:2223-33

4 Costanzo S, Di Castelnuovo A, Donati MB, et al. Alcohol consumption and mortality in patients with cardiovascular disease: a meta-analysis. J Am Coll Cardiol 2010;55:1339-47.

5 O'Donnell MJ, Xavier D, Liu L, et al. Risk factors for ischaemic and intracerebral haemorrhagic stroke in 22 countries (the INTERSTROKE study): a case-control study. Lancet 2010;376:112-23.

6 Reynolds K, Lewis LB, Nolen JDL, et al. Alcohol consumption and risk of stroke: a meta-analysis. JAMA 2003;289:579-88.

7 Kodama S, Saito K, Tanaka S, et al. Alcohol consumption and risk of atrial fibrillation: a meta-analysis. J Am Coll Cardiol 2011;57:427-36.

8 Pisters R, Lane DA, Nieuwlaat R, et al. A novel user-friendly score (HAS-BLED) to assess 1-year risk of major bleeding in patients with atrial fibrillation: the Euro Heart Survey. Chest 2010;138:1093-100.

9 Gage BF, Yan Y, Milligan PE, et al. Clinical classification schemes for predicting hemorrhage: results from the National Registry of Atrial Fibrillation (NRAF). Am Heart I 2006;151:713-19.

10 Shireman TI, Mahnken JD, Howard PA, et al. Development of a contemporary bleeding risk model for elderly warfarin recipients. Chest 2006;130:1390-6.

11 Lip GYH, Frison L, Halperin JL, et al. Identifying patients at high risk for stroke despite anticoagulation: a comparison of contemporary stroke risk stratification schemes in an anticoagulated atrial fibrillation cohort. Stroke 2010;41:2731-8.

12 Hart RG, Pearce LA, McBride $R$, et al. Factors associated with ischemic stroke during aspirin therapy in atrial fibrillation: analysis of 2012 participants in the SPAF I-III clinical trials. Stroke 1999;30:1223-9.

13 Mukamal KJ, Psaty BM, Rautaharju PM, et al. Alcohol consumption and risk and prognosis of atrial fibrillation among older adults: the Cardiovascular Health Study. Am Heart J 2007;153:260-6.

14 Tjønneland A, Olsen A, Boll K, et al. Study design, exposure variables, and socioeconomic determinants of participation in Diet, Cancer and Health: a population-based prospective cohort study of 57,053 men and women in Denmark. Scand I Public Health 2007;35:432-41.
15 Thygesen LC, Daasnes C, Thaulow I, et al. Introduction to Danish (nationwide) registers on health and social issues: structure, access, legislation, and archiving. Scand J Public Health 2011:39:12-16.

16 Overvad K, Tjønneland A, Haraldsdóttir J, et al. Development of a semiquantitative food frequency questionnaire to assess food, energy and nutrient intake in Denmark. Int J Epidemiol 1991;20:900-5.

17 Tjønneland A, Overvad K, Haraldsdóttir J, et al. Validation of a semiquantitative food frequency questionnaire developed in Denmark. Int J Epidemiol 1991;20:906-12.

18 Olesen JB, Lip GYH, Hansen ML, et al. Validation of risk stratification schemes for predicting stroke and thromboembolism in patients with atrial fibrillation: nationwide cohort study. BMJ 2011;342:d124.

19 Camm AJ, Lip GYH, De Caterina R, et al. 2012 focused update of the ESC Guidelines for the management of atrial fibrillation: an update of the 2010 ESC Guidelines for the management of atrial fibrillation. Eur Heart J 2012;33:2719-47.

20 You JJ, Singer DE, Howard PA, et al. Antithrombotic therapy for atrial fibrillation: antithrombotic therapy and prevention of thrombosis, 9th ed: American College of Chest Physicians evidence-based clinical practice guidelines. Chest 2012;141 e5315-575S.

21 Camm JA, Kirchhof P, Lip GYH, et al. Guidelines for the management of atrial fibrillation: the Task Force for the Management of Atrial Fibrillation of the European Society of Cardiology (ESC). Eur Heart J 2010;31:2369-429.

22 Rix TA, Riahi S, Overvad K, et al. Validity of the diagnoses atrial fibrillation and atrial flutter in a Danish patient registry. Scand Cardiovasc J 2012;46:149-53.

23 Tsubono Y, Yamada S, Nishino Y, et al. Choice of comparison group in assessing the health effects of moderate alcohol consumption. JAMA 2001;286:1177-8.

24 Hart RG, Pearce LA, Aguilar MI. Meta-analysis: antithrombotic therapy to prevent stroke in patients who have nonvalvular atrial fibrillation. Ann Intern Med 2007;146:857-67.

25 Larsen TB, Lip GYH, Skjøth F, et al. Added predictive ability of the CHA2DS2VASc risk score for stroke and death in patients with atrial fibrillation: the prospective Danish Diet, Cancer, and Health cohort study. Circ Cardiovasc Qual Outcomes 2012;5:335-42.

26 Granger $\mathrm{CB}$, Alexander JH, McMurray JJ, et al. Apixaban versus warfarin in patients with atrial fibrillation. N Engl I Med 2011;365:981-92.

27 Patel MR, Mahaffey KW, Garg J, et al. Rivaroxaban versus warfarin in nonvalvular atrial fibrillation. N Engl J Med 2011;365:883-91.

28 Connolly SJ, Ezekowitz MD, Yusuf S, et al. Dabigatran versus warfarin in patients with atrial fibrillation. N Engl I Med 2009;361:1139-51. 